\title{
Chromosomal and Plasmid-Encoded Factors of Shigella flexneri Induce Secretogenic Activity Ex Vivo
}

\author{
Christina Faherty ${ }^{19}$, Jill M. Harper ${ }^{29}$, Terez Shea-Donohue ${ }^{2}$, Eileen M. Barry ${ }^{1}$, James B. Kaper ${ }^{3}$, \\ Alessio Fasano $^{2 *}$, James P. Nataro ${ }^{1 *}$
}

1 Department of Medicine, Center for Vaccine Development, University of Maryland School of Medicine, Baltimore, Maryland, United States of America, 2 Mucosal Biology Research Center, University of Maryland School of Medicine, Baltimore, Maryland, United States of America, 3 Department of Microbiology and Immunology, University of Maryland School of Medicine, Baltimore, Maryland, United States of America

\begin{abstract}
Shigella flexneri is a Gram-negative, facultative intracellular pathogen that causes millions of cases of watery or bloody diarrhea annually, resulting in significant global mortality. Watery diarrhea is thought to arise in the jejunum, and subsequent bloody diarrhea occurs as a result of invasion of the colonic epithelium. Previous literature has demonstrated that Shigella encodes enterotoxins, both chromosomally and on the 220 kilobase virulence plasmid. The Shigella Enterotoxins 1 and 2 (ShET1 and ShET2) have been shown to increase water accumulation in the rabbit ileal loop model. In addition, these toxins increase the short circuit current in rabbit tissue mounted in Ussing chambers, which is a model for the ion exchange that occurs during watery diarrhea. In this study, we sought to validate the use of mouse jejunum in Ussing chamber as an alternative, more versatile model to study bacterial pathogenesis. In the process, we also identified enterotoxins in addition to ShET1 and ShET2 encoded by S. flexneri. Through analysis of proteins secreted from wildtype bacteria and various deletion mutants, we have identified four factors responsible for enterotoxin activity: ShET1 and Pic, which are encoded on the chromosome; ShET2 (encoded by sen or ospD3), which requires the type-III secretion system for secretion; and SepA, an additional factor encoded on the virulence plasmid. The use of mouse jejunum serves as a reliable and reproducible model to identify the enterotoxins elaborated by enteric bacteria. Moreover, the identification of all Shigella proteins responsible for enterotoxin activity is vital to our understanding of Shigella pathogenicity and to our success in developing safe and effective vaccine candidates.
\end{abstract}

Citation: Faherty C, Harper JM, Shea-Donohue T, Barry EM, Kaper JB, et al. (2012) Chromosomal and Plasmid-Encoded Factors of Shigella flexneri Induce Secretogenic Activity Ex Vivo. PLoS ONE 7(11): e49980. doi:10.1371/journal.pone.0049980

Editor: Nicholas J. Mantis, Wadsworth Center, New York State Dept. Health, United States of America

Received September 8, 2012; Accepted October 15, 2012; Published November 16, 2012

Copyright: (c) 2012 Faherty et al. This is an open-access article distributed under the terms of the Creative Commons Attribution License, which permits unrestricted use, distribution, and reproduction in any medium, provided the original author and source are credited.

Funding: This work was supported by NIH 5U19AI090873 and NIH R01-AI059223. The Center for Vaccine Development awarded the T32 Vaccinology Fellowship Al07524 to C.S.F. The funders had no role in study design, data collection and analysis, decision to publish, or preparation of the manuscript.

Competing Interests: The authors have declared that no competing interests exist.

*E-mail: afasano@mbrc.umaryland.edu

a Current address: Department of Pediatrics, University of Virginia School of Medicine, Charlottesville, Virginia, United States of America

9 These authors contributed equally to this work.

\section{Introduction}

Enteric infections resulting in diarrhea are prevalent throughout the developed and developing world. Children with frequent diarrheal episodes are at risk for life-long physical and mental defects [1]. While viruses and parasites cause a significant amount of diarrheal disease, bacterial pathogens, including the pathogenic strains of Escherichia coli, Shigella, and Salmonella, are a leading cause of diarrheal disease in the developing world [2]. Preventive and prophylactic measures have been difficult to develop and implement, making diarrheagenic bacteria a nuisance to public health and detrimental to the health of the affected populations. The lack of available animal models has been a hindrance to the process of vaccine and drug development.

Shigella species comprise a leading cause of diarrhea-associated morbidity and mortality in children under the age of five years in developing countries [3]. This age group is particularly susceptible to the effects of acute and chronic infections, although Shigella is clinically important in all age groups in the developing world. In addition to dehydration and the inability to absorb nutrients, children who are continuously exposed to pathogenic bacteria suffer from both physical and developmental milestone delays [1]. Stools from these children frequently contain multiple infectious organisms; however, Shigella flexneri 2a is the most prominent bacterial pathogen isolated from children suffering from diarrhea between the ages of 2 and 5 years [3]. Although the invasion process has been thoroughly dissected, the mechanisms by which Shigella induces secretory diarrhea are less defined. Like many other pathogenic bacteria, S. flexneri 2a possesses a type-III secretion system (T3SS) that produces several proteins required for invasion of intestinal cells. At least one T3SS effector has been identified as a putative toxin [4,5]. Most, if not all, pathogenic bacteria elaborate one or more toxins during the course of infection. These toxins have diverse activities that include, but are not limited to, colonization, proteolytic activity, cellular toxicity, and increasing intestinal secretion [6,7].

To date few studies have addressed the effects of extracellularly secreted bacterial products during $S$. flexneri 2a infection. Studies performed in the 1990s described two toxins produced by $S$. flexneri 2a, Shigella enterotoxin 1 (ShET1) and Shigella enterotoxin 2 
(ShET2), that contribute to secretory activity in rabbit intestine in vivo and in vitro $[4,8,9]$. An epidemiological study of 383 Shigella isolates found that ShET1 was present in $30 \%$ of all Shigella isolates while ShET2 was present in $80 \%$ of isolates. Watery diarrhea is present in almost all shigellosis cases, and typically proceeds to dysentery. In fact in mild infections, watery diarrhea is the only clinical manifestation. [10,11]. ShET1 is encoded on the chromosome in the she pathogenicity island (PAI), and is only found in S. flexneri [8]. ShET2 is a T3SS effector encoded on the $220 \mathrm{~kb}$ invasion plasmid [5]. Previous studies reported the ability of bacterial supernatants prepared in iron-depleted medium to increase secretion in both Ussing chambers and the rabbit ileal loop model $[4,8,9]$. It was theorized that the secretion of ShET1 and ShET2 in the upper intestine (e.g. jejunum) would increase fluid secretion which, in turn, would facilitate the transit of bacteria to the colon, the site of invasion. This observation accounts for the watery diarrhea, as opposed to the scant bloody diarrhea, that occurs as a result of PMN infiltration at the site of invasion [12].

The overarching goal of this study was to validate mouse tissue as a useful model of intestinal secretion caused by exposure to secreted toxins. We also aimed at identifying all secreted factors that contribute to jejunal secretion to better define the mechanism of Shigella-induced diarrhea. In vivo intestinal secretion as a result of $S$. flexneri infection is difficult to study as the pathogen will only infect non-human primates at the natural site of infection [13]. Ussing chambers are a well-established system to define secretion from intestinal tissue ex vivo, and provide an alternative to in vivo studies [14]. Previous studies defined the response of rabbit jejunum to secreted Shigella toxins and recombinant proteins in the Ussing chambers $[4,8,9]$. We extended this secretory model to include mouse jejunum, which has proven to respond similarly to rabbit tissue. Herein we demonstrate the utility of mouse intestine as an affordable and informative model for defining secretory activity of bacterial toxins. Our study implicates both chromosomal and plasmid-encoded toxins as contributors to intestinal secretion caused by $S$. flexneri 2a. Identification of all of the enterotoxins encoded by $S$. flexneri is vital to our understanding of Shigella pathogenesis and may lead to targets suitable for drug and vaccine development.

\section{Materials and Methods}

\section{Strains and Growth Conditions}

Table 1 lists the bacterial strains and plasmids used in this study. Table 2 lists the enterotoxins expressed for each bacterial strain used in this study. Bacteria were routinely cultured at $37^{\circ} \mathrm{C}$ either in Luria-Bertani broth with aeration, or on tryptic soy broth plates with $1.5 \%$ agar and $0.025 \%$ Congo Red (CR; Sigma). Antibiotics were used at the following concentrations: kanamycin, $50 \mu \mathrm{g} / \mathrm{ml}$; streptomycin, $50 \mu \mathrm{g} / \mathrm{ml}$; and chloramphenicol, $5 \mu \mathrm{g} / \mathrm{ml}$. Guanine supplementation $(0.005 \%$, Sigma) was used for strains harboring the $\triangle$ guaBA mutations (strain 1208S).

\section{Mutant Construction}

Strain 1377 ( $\Delta$ pic and $\Delta$ set $1 \mathrm{AB})$ was constructed using a modification of the $\lambda$ red linear recombination method [15] in which the entire $p i c / s e t 1 \mathrm{AB}$ region was replaced with a kanamycin/sacB cassette. Selection for the mutation was performed using kanamycin. Sucrose sensitivity and PCR analysis verified that the mutation was achieved at the appropriate site. Strain 1380 is a spontaneous T3SS mutant, and was selected for by red/white colony screenings on CR plates. To construct 1522, the kanamycin/sacB cassette from 1377 was removed by a second linear recombination step in which the wildtype pic/set $1 \mathrm{AB}$ locus was replaced back into the region of interest [16]. To construct 1542 the pic/set $1 \mathrm{AB}$ locus with four point mutations to terminate transcription of set $1 \mathrm{~A}$ and set $1 \mathrm{~B}$ while maintaining transcription of pic was replaced back into the region of interest [16]. 1522 and 1542 were selected via sucrose resistance and screened for kanamycin sensitivity. DNA sequencing confirmed restoration of the wildtype or point mutation locus.

Strain BS796 ( $\operatorname{ospD3})$ was constructed using the $\lambda$ red linear recombination method as previously described [15]. PCR was used to amplify a chloramphenicol resistance cassette gene (cat) from pKD3 (Table 1) which had $5^{\prime}$ and $3^{\prime}$ overhangs identical to the $5^{\prime}$ and $3^{\prime}$ regions of $\operatorname{spD} 3$. The sequence of the forward primer was $5^{\prime}$ - TGA AAG GAA TAT ATA CAT ATG CGA TCA GTA AAT TTA ATC CGA TCA AGG CAT ATG AAT ACG TCG TTA GTT CG $-3^{\prime}$ and the sequence of the reverse primer was $5^{\prime}$ - ATC ACG AGG AGA TAA TAT TGA GGT TTT TAT ATT CTT CAT AAT TT CTA GTG TGT AGG CTG GAG CTG CTT C $-3^{\prime}$. Chloramphenicol resistant recombinants were purified and screened via PCR using primers that annealed to unique regions upstream and downstream of ospD3 to detect the size difference due to the insertion of the chloramphenicol cassette. For this PCR reaction, the forward primer sequence was $5^{\prime}$ - GCG ATC AAT CAT CCA AAG GG $-3^{\prime}$ and the reverse primer sequence was 5' - CGG AGG TAA CAG ACG AGA CG -3'. This mutant, in the BS766 background, was used as the donor strain for transduction of $2457 \mathrm{~T}$ (the recipient) using P1L4 [17], and selection for chloramphenicol resistance generated BS796. The mutation was verified by PCR.

\section{Animals Used in this Study}

Female New Zealand White (NZW) rabbits were purchased from Charles River. Rabbits weighing between 2.3 and $3 \mathrm{~kg}$ were used in Ussing chamber experiments. Female C57BL/6 mice were purchased from the National Cancer Institute (Frederick, MD) and were used for Ussing experiments between 6 and 14 weeks of age.

\section{Preparation of Bacterial Supernatants}

Samples were prepared as follows for use in Ussing studies. Overnight cultures of bacteria were subcultured into $600 \mathrm{ml}$ Luria-Bertani Broth (LB) with the appropriate antibiotic selection or nutrient supplementation required for each strain of $S$. flexneri (see strains and growth conditions). Cultures were grown at $37^{\circ} \mathrm{C}$ with shaking until the optical density $\left(\mathrm{OD}_{600}\right)$ reached 1.0. The bacteria were then pelleted by centrifugation at 5,000 rpm for 10 minutes at $4^{\circ} \mathrm{C}$. Culture supernatants were subsequently filtersterilized with a $0.45 \mu \mathrm{m}$ vacuum filtration unit (Millipore) and stored at $-20^{\circ} \mathrm{C}$. The stored supernatants were thawed at $4^{\circ} \mathrm{C}$ and concentrated using the Centricon Plus-70 centrifugal filter devices with a molecular weight cutoff of $30 \mathrm{kD}$ (Millipore), according to manufacturer's instructions. Concentrated proteins (averaging $30 \mu \mathrm{g} / \mu \mathrm{l})$ were resuspended in $1 \mathrm{X}$ PBS to a final volume of $2 \mathrm{ml}$, and samples were stored at $-80^{\circ} \mathrm{C}$ in $500 \mu \mathrm{l}$ aliquots.

\section{Preparation of Iron-depleted LB}

In order to deplete the iron from LB, fifty grams of Chelex-100 resin (Bio-Rad) was added to $1 \mathrm{~L}$ of $\mathrm{LB}$ according to manufacturer's instructions and stirred for 2 hours, after which the solution was filtered through a $0.45 \mu \mathrm{M}$ vacuum filter unit with receiver bottle (Millipore). $\mathrm{MgSO}_{4}$ was added to a final concentration of $2 \mathrm{mM}$ and $\mathrm{CaCl}_{2}$ was added to a final concentration of $0.1 \mathrm{mM}$. All solutions were prepared with iron-free water in iron-free containers. The $\mathrm{pH}$ of the solution was adjusted to 7.4 with 
Table 1. Strains and plasmids used in this study.

\begin{tabular}{|c|c|c|}
\hline Name & Description & Reference \\
\hline \multicolumn{3}{|l|}{ Strains } \\
\hline $2457 T$ & Wildtype S. flexneri 2a & [36] \\
\hline BS103 & Plasmid-cured $2457 \mathrm{~T}$ & [37] \\
\hline BS652 & 2457T/spa47::aadA & [38] \\
\hline 1377 & 2457T/pic/set1AB::aph-3 & [16] \\
\hline 1380 & 1377/type-III secretion system mutant & This study \\
\hline 1522 & 1377/pic $/$ set1AB ${ }^{+}$ & {$[16]$} \\
\hline 1542 & 1377/pic ${ }^{+}$ & [16] \\
\hline BS796 & 2457T/ospD3.:.cat & ATM; this study \\
\hline \multirow[t]{2}{*}{12085} & $2457 \mathrm{~T} / \Delta g u a B A+\Delta$ set $1 \mathrm{AB}+\Delta o s p D 3$ & [39] \\
\hline & $2457 \mathrm{~T} / \Delta \operatorname{sep} A$ & BAM \\
\hline E. coli HS & Commensal E. coli; $\mathrm{Nal}^{r}$ & [23] \\
\hline \multicolumn{3}{|c|}{ Mutant Construction } \\
\hline \multicolumn{3}{|l|}{ Strains } \\
\hline BS766 & $2457 \mathrm{~T}$ transformed with pKM208 & [38] \\
\hline \multicolumn{3}{|l|}{ Plasmids } \\
\hline pKD3 & oriR6K, bla, cat & [15] \\
\hline pKM208 & Temperature-sensitive red-, gam-, lacl-expressing plasmid driven by $P_{T a c}$ promoter, bla & [15] \\
\hline
\end{tabular}

concentrated HCl. The iron-free LB was then filtered a second time through a $0.45 \mu \mathrm{M}$ filter to ensure sterility. Medium was used immediately or stored at $4^{\circ} \mathrm{C}$ until use.

\section{Ussing Chamber Studies}

Assessment of secretory activity in response to secreted bacterial proteins was performed using jejunum mucosae stripped of muscle and mounted in Ussing chambers [18]. For rabbit jejunum $0.5206 \mathrm{~cm}^{2}$ tissue was exposed for analysis in the Ussing chambers, while $0.1256 \mathrm{~cm}^{2}$ tissue was exposed for mouse jejunum. While in the Ussing chambers, the tissue was short circuited every $50 \mathrm{~s}$ at $1 \mathrm{~V}$ (World Precision Instruments DVC, 1000 voltage clamp) and the short circuit current (Isc) was monitored continuously. The potential difference was measured

Table 2. Toxins expressed in the Shigella flexneri strains analyzed.

\begin{tabular}{lllll}
\hline & & & & \\
\hline Strain & ShET1 & Pic & ShET2 & SepA \\
\hline $2457 \mathrm{~T}$ & + & + & + & + \\
BS103 & + & + & - & - \\
BS652 & + & + & - & + \\
1377 & - & - & + & + \\
1380 & - & - & - & + \\
1522 & + & + & + & + \\
1542 & - & + & + & + \\
BS796 & + & + & - & + \\
$1208 S$ & - & - & - & + \\
$\Delta$ sepA & + & + & + & - \\
E. coli HS & - & - & - & - \\
\hline doi:10.1371/journal.pone.0049980.t002 & & &
\end{tabular}

using agar-salt bridges and electrodes. After equilibration of $0.126 \mathrm{~cm}^{2}$ of tissue in $10 \mathrm{ml}$ of Krebs' buffer $(118 \mathrm{mM} \mathrm{NaCl}$, $4.7 \mathrm{mM} \mathrm{KCl}, 1.2 \mathrm{mM}$ KH2PO4, $1.2 \mathrm{mM} \mathrm{MgSO} 4,4.2 \mathrm{mM}$ NaHCO3, 2 mM CaCl2, 10 mM glucose, 200 mM sulphinpyrazone and $10 \mathrm{mM}$ Hepes, $\mathrm{pH}$ 7.4), a test for glucose absorption was conducted to assess the responsiveness of the tissue. To test the function of the sodium linked glucose transporter and, therefore, the integrity of the tissue, $20 \mathrm{mM}$ glucose was added to the mucosal chamber while an iso-osmotic solution of mannitol was added to the serosal chamber; and Isc determined. The tissues were washed and allowed to equilibrate once more, after which $400 \mu \mathrm{l}$ of concentrated secreted Shigella proteins were added to the mucosal chamber and $400 \mu \mathrm{l}$ of PBS was added to the serosal chamber. Isc was measured for at least 30 minutes post-toxin addition; the change in Isc $(\Delta \mathrm{Isc})$ reflects the maximum change during this time course. Responses from two tissue segments exposed to secreted proteins from one mouse were averaged to yield a mean response per animal. Each sample was tested on a minimum of four animals. For every experiment, secreted proteins from wildtype $2457 \mathrm{~T}$ served as a positive control. Values are expressed as mean \pm standard error of the mean (SEM).

\section{Congo Red Secretion Assay}

The Congo red (CR) secretion assay was used to identify proteins secreted by the bacteria through the T3SS, and was performed as previously described [19]. Briefly, bacteria were grown to mid-log phase, resuspended in IX PBS, and CR was added to a final concentration of $30 \mu \mathrm{g} / \mathrm{ml}$. The bacteria were incubated at $37^{\circ} \mathrm{C}$ for 1 hour. After incubation, the bacteria were pelleted by centrifugation, and the supernatant was collected and filtered through a $0.22-\mu \mathrm{m}$-pore filter and stored at $-20^{\circ} \mathrm{C}$. The proteins in the supernatant represent the secreted proteins and were concentrated by trichloroacetic acid (TCA) precipitation. TCA pellets were resuspended in $50 \mu \mathrm{l}$ sodium dodecyl sulfate (SDS) loading buffer for protein analysis and stored at $-20^{\circ} \mathrm{C}$. The 
bacterial pellets, representing the non-secreted proteins, were resuspended in $500 \mu \mathrm{l}$ of SDS loading buffer and stored at $-20^{\circ} \mathrm{C}$.

\section{Protein and Western Blot Analysis}

For total protein analysis each sample was resolved by SDSpolyacrylamide gel electrophoresis (PAGE), and Coomassie blue stain was used to visualize total protein. For Western blot analysis proteins were transferred to a PVDF membrane and blocked with $10 \%$ dry milk in $1 \mathrm{X}$ PBS. IpaB was detected with mouse monoclonal anti-IpaB antibody (1:20,000 dilution) [20]. After washing, Alexa Fluor 700 goat anti-mouse immunoglobulin G $(\mathrm{H}+\mathrm{L})$ antibody (Molecular Probes) was added at a 1:3,000 concentration in PBS-Tween with 10\% dry milk for $1 \mathrm{~h}$. All washes of the PVDF membrane were performed with 1X PBST for 5 minutes at room temperature. Protein gels and Western blots were scanned using the Odyssey infrared detection system (LiCor).

\section{Statistical Analysis}

Two-tailed Student's t-tests or analysis of variance (ANOVA) were used to assess significance with $\mathrm{p}<0.05$ considered to be significant.

\section{Ethics Statement}

This study was carried out in strict accordance with the recommendations in the Guide for the Care and Use of Laboratory Animals of the National Institutes of Health. The protocol was approved by the Institutional Animal Care and Use Committee (IACUC) at the University of Maryland at Baltimore (protocol \#0111044). All efforts were made to minimize suffering during euthanasia of the mice.

\section{Results}

\section{Iron-depletion of Medium does not Affect Enterotoxic Activity}

Previous studies with enteroinvasive E. coli required iron depletion in order to detect an enterotoxin effect in the rabbit ileal loop model and increases in Isc in the Ussing chambers [21]. These iron-depleted conditions were continued with studies for $S$. flexneri; however, it was never determined if iron-depletion was required to induce enterotoxin expression in $S$. flexneri. In this study, we tested whether enterotoxic activity of $S$. flexneri $2457 \mathrm{~T}$ was only apparent after preparation with iron-depleted media. Cultures were prepared with and without Chelex-100 resin, and the culture supernatants were processed as described in the Materials and Methods. Supernatants from 2457T cultures prepared with and without the iron chelator both resulted in a significant increase in Isc, and no significant difference was detected between the two conditions on rabbit tissue (Figure 1). The Vibrio cholerae toxin served as the positive control [22] whereas PBS and supernatants derived from the commensal E. coli HS strain, which does not produce any enterotoxic activity [23], were employed as the negative controls. These observations were confirmed in experiments with mouse tissue (data not shown). As a result of these experiments, we maintained normal growth conditions, without iron depletion, for analysis of the S. flexneri deletion mutants.

\section{The Plasmid-cured Strain and a Type-III Secretion System Mutant Identify Loci of Putative Enterotoxin Genes}

In order to corroborate and expand previous studies on the location (chromosome, virulence plasmid, or both) of genes encoding enterotoxic factors, we compared the plasmid-cured strain of S. flexneri (strain BS103) and a T3SS mutant (strain BS652) to wildtype 2457T. BS652 harbors a deletion in spa47, which encodes the ATPase that drives the T3SS. This mutation results in assembly of the T3SS apparatus but prevents secretion of T3SS effector proteins [24,25]. Therefore, proteins encoded on the virulence plasmid that do not require the T3SS for secretion are still present in the culture supernatants. E. coli HS served as a negative control for toxigenic activity. The protein profile of the concentrated supernatants for each strain is provided in Figure 2. High molecular weight proteins are encoded on both the chromosome and virulence plasmid, and represent the large autotransporter proteins encoded by the bacteria [26,27,28,29]. The smaller proteins present in the $2457 \mathrm{~T}$ supernatants represent the effector proteins secreted by the T3SS [30].

Analysis of the concentrated supernatants on rabbit tissue in the Ussing chambers demonstrated that 2457T consistently induced an increase in Isc of approximately $100 \mu \mathrm{A} / \mathrm{cm}^{2}$ while the enterotoxic signal triggered by strain BS103 was significantly lower compared to $2457 \mathrm{~T}$, with a consistent Isc of approximately $40 \mu \mathrm{A} / \mathrm{cm}^{2}$ (Figure 3A). The data suggested that the chromosome harbors genes encoding enterotoxins. The $\Delta \mathrm{Isc}$ of approximately $60 \mu \mathrm{A} / \mathrm{cm}^{2}$ caused by strain BS652 was significantly lower than that induced by $2457 \mathrm{~T}$ supernatants, but was also significantly higher than BS103 (Figure 3A). Since BS652 has a defective T3SS, the data indicated that there are two types of toxin contributions from the virulence plasmid. The first type is an effector secreted through the T3SS and the second type does not require the T3SS to be secreted. Based on the annotated sequence of the virulence plasmid and the appearance of supernatants on protein gel electrophoresis, we hypothesized that a high molecular weight autotransporter encoded on the plasmid would contribute to enterotoxin activity (see below). To develop a more practical and efficient ex vivo assay, we also performed analysis of the same strains on tissue isolated from mice. The supernatants were prepared and applied to the mouse jejunal tissue in the same manner as described for the rabbit jejunum. We found that 2457T, BS103, and BS652 induced similar changes in Isc in the mouse tissue as in the rabbit tissue (Figure 3B). Given the benefits of utilizing mouse tissue, we proceeded with mouse jejunum for the remainder of the experiments with $2457 \mathrm{~T}$ supernatants serving as the positive control.

\section{Identification of the Genes Encoding the Chromosomal Enterotoxin}

In order to identify the toxins encoded on the chromosome, we first analyzed a deletion mutant of the previously implicated set $1 \mathrm{~A}$ and set1B genes, which encode ShET1 (strain 1377). Studies have demonstrated that in S. flexneri 2a the pic gene is encoded on the opposite strand of DNA from that encoding the set $1 \mathrm{AB}$ region [16]. To ensure that all potential enterotoxic activity was removed from this region, mutant 1377 had the entire pic/set $1 \mathrm{AB}$ region deleted. Strain 1377 produced a change in Isc of approximately $40 \mu \mathrm{A} / \mathrm{cm}^{2}$ (Figure 4). Complementation of strain 1377 with the wildtype pic/set $1 \mathrm{AB}$ locus replaced in strain 1522 restores enterotoxic activity to the same level as $2457 \mathrm{~T}$ (Figure 4). The data therefore indicate that the chromosomal enterotoxic contribution arises from the ShET1/Pic region. Interestingly, when 1377 was complemented with pic alone in strain 1542, enterotoxic activity was restored to $2457 \mathrm{~T}$ levels. In order to achieve complementation with pic alone, the locus was replaced with a cassette containing four point mutations that introduced stop codons in the set1A and set $1 \mathrm{~B}$ genes while maintaining the appropriate codons for pic. These data suggested that Pic is the 


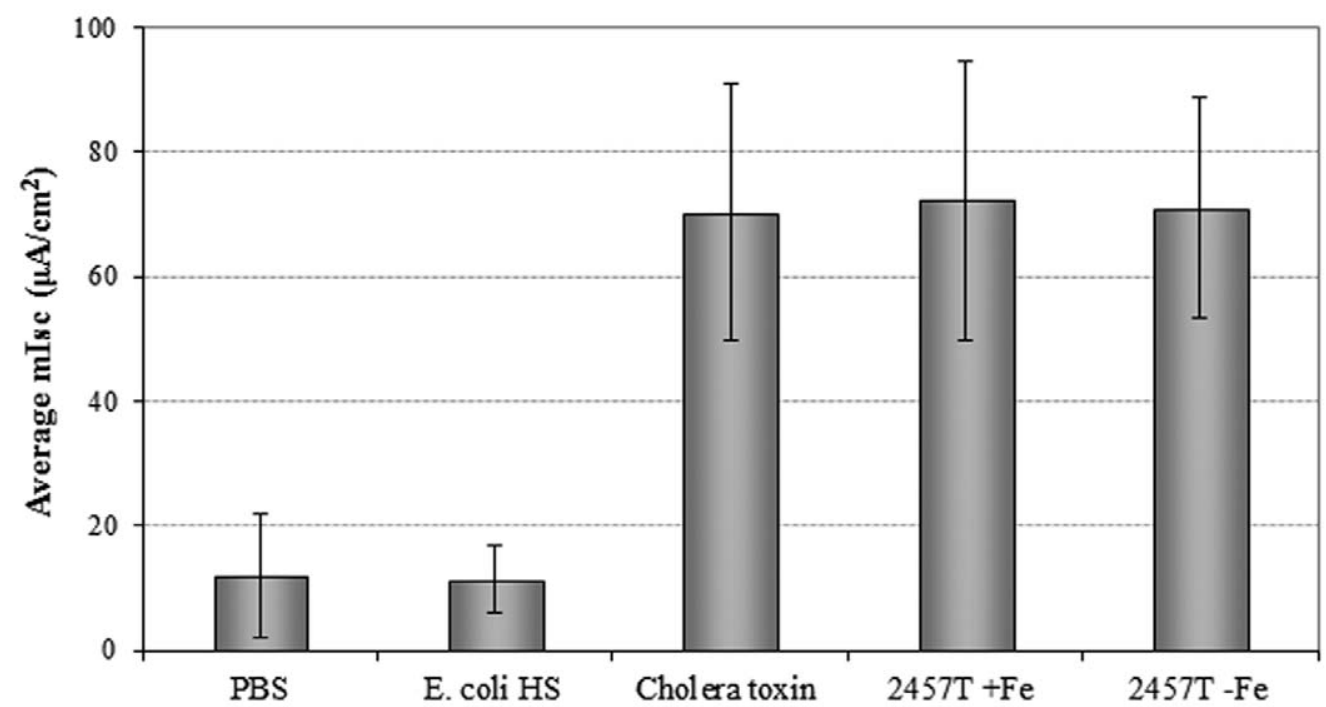

Figure 1. Iron depletion is not required for $S$. flexneri enterotoxin activity. The average change in short circuit current $(\mu \mathrm{A} / \mathrm{cm} 2)$ is plotted $+/$ - the standard error for wildtype S. flexneri 2a strain $2457 \mathrm{~T}$ with iron ( $+\mathrm{Fe}$ ) and without iron (-Fe). There is no significant difference between the two conditions. PBS and supernatant from E. coli HS served as negative controls while $40 \mu \mathrm{g}$ of Vibrio cholerae toxin served as a positive control for inducing a change in Isc.

doi:10.1371/journal.pone.0049980.g001

enterotoxin producing the greater contribution. Expression of set1 $\mathrm{AB}$ alone, however, was sufficient to induce a significant

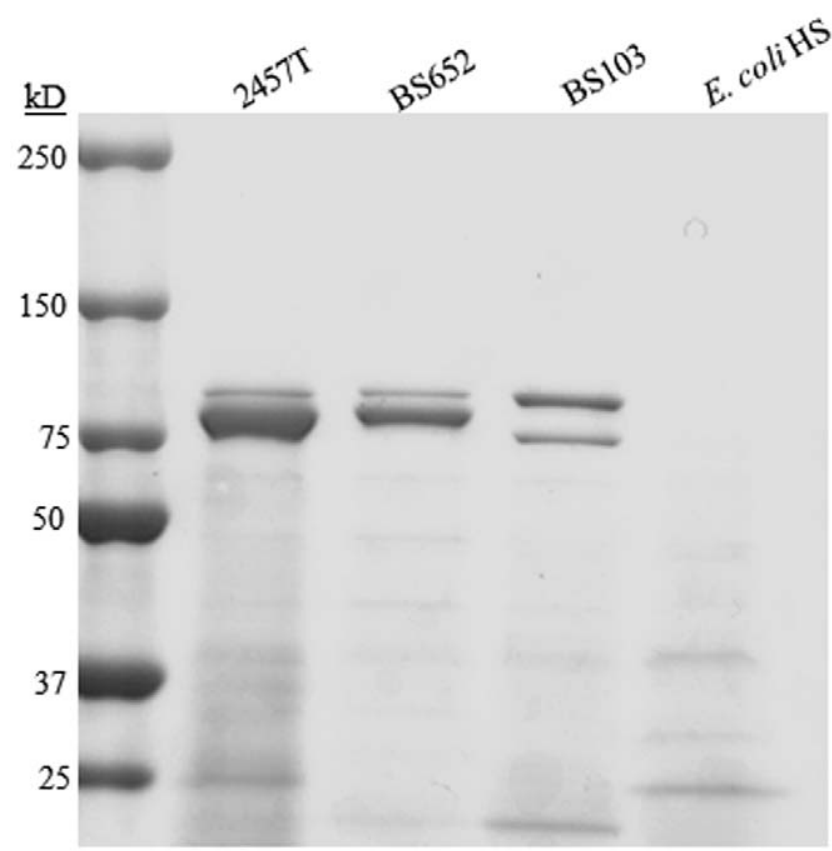

Figure 2. Coomassie-stained protein gel of the secreted proteins obtained from each strain of bacteria after growth and concentration of cultural supernatants. Wildtype S. flexneri $2 \mathrm{a}$ strain $2457 \mathrm{~T}$ secretes the autotransporter proteins localized around $100 \mathrm{kD}$ in addition to other type-III secretion system effector proteins at lower molecular weights. Strain BS652 has a defective T3SS but is still able to secrete the autotransporter SepA that is encoded on the virulence plasmid. Strain BS103 is the virulence plasmid-cured and therefore secrets only the chromosomal autotransporters. The negative control E. coli $\mathrm{HS}$ commensal strain secretes a minimal amount of protein.

doi:10.1371/journal.pone.0049980.g002 change in Isc $[8,9]$. Therefore, we conclude that both Pic and ShET1 confer enterotoxic activity. Our data suggest that ShET1 and Pic are the chromosomally-encoded enterotoxins expressed under the culture conditions used in our experiments.

\section{Identification of the Genes Encoding the Virulence Plasmid Enterotoxins}

Given that strain BS652 is a T3SS mutant and that ShET2 has been identified as a T3SS effector protein, we next analyzed strain BS796, which harbors a mutation in sen (also called ospD3), the gene encoding ShET2. As shown in figure 5, a mutation in ShET2 resulted in approximately the same $\Delta \mathrm{Isc}$ as a mutant with a defective T3SS (strain BS652), suggesting that the enterotoxin secreted by the T3SS was in fact ShET2.

\section{Identification of Additional Enterotoxins Encoded by Shigella flexneri}

The $\Delta \mathrm{Isc}$ induced by strains 1380 and $1208 \mathrm{~S}$ suggested that more enterotoxins were present in addition to Pic, ShET1, and ShET2. 1208S harbors a double mutation in ShET1 and ShET2. Strain 1380 is a spontaneous T3SS mutant of 1377 that was isolated by looking for white colonies on CR plates. PCR analysis for the ospC1 to ospD3 coding region $(3.7 \mathrm{~kb})$ revealed that 1380 still harbored the virulence plasmid (Figure 6A), and therefore was not a plasmid-cured strain of 1377 . To verify that 1380 had a defective T3SS, a CR secretion assay was performed to ensure T3-secreted proteins were not present in supernatants. As shown in figure $6 \mathrm{~B}$, T3SS-specific secretion was significantly reduced in 1380, similar to the response in the 4 spa47 mutant (strain BS652). To confirm that secretion was T3SS-dependent, we performed Western blot analysis to detect the T3SS effector IpaB (Figure 6C); as expected, 1380 did not secrete IpaB. Therefore, we concluded that 1380 culture supernatants were deficient for Pic and ShET 1 (due to the mutation), and ShET2 due to the lack of T3 effector secretion.

Strains 1208 S and 1380 were analyzed using Ussing chambers to determine if some enterotoxic activity was retained despite removal or inadequate secretion of ShET1, Pic and, ShET2. 


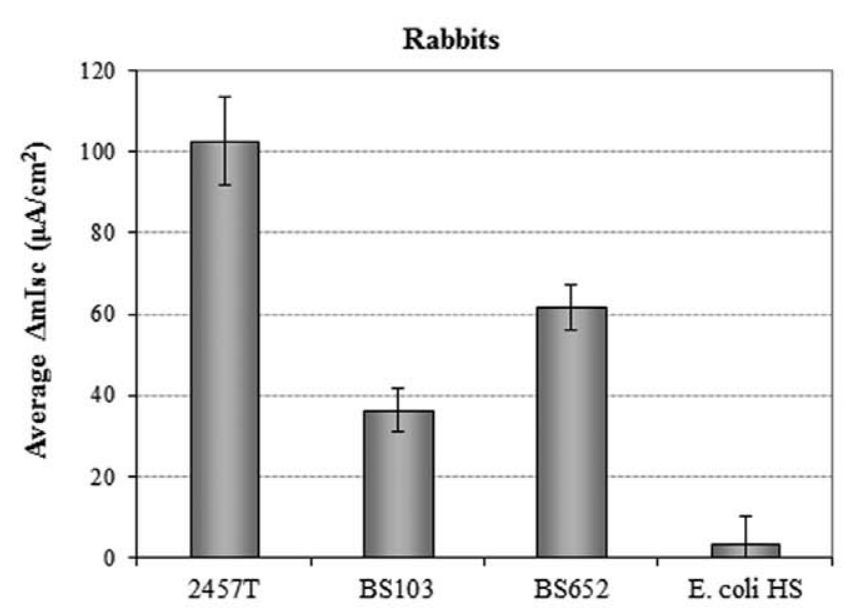

B

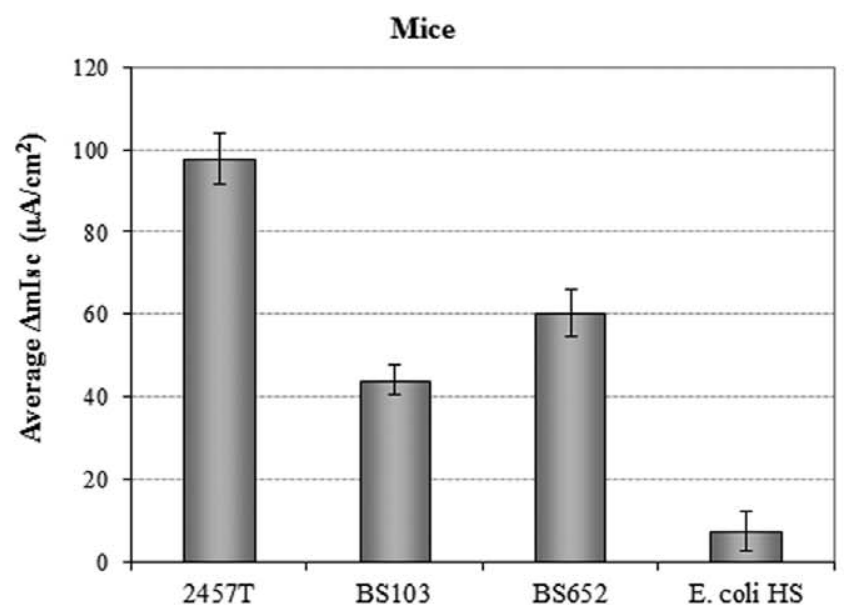

Figure 3. The $S$. flexneri chromosome and virulence plasmid encode enterotoxins that increase Isc in both rabbit and mouse tissue. The average change in short circuit current $\left(\mathrm{m} \Delta \mathrm{lsc}, \mu \mathrm{A} / \mathrm{cm}^{2}\right)$ is plotted $+/-$ the standard error for wildtype S. flexneri strain 2457T, the virulence plasmid-cured strain BS103, and the $\Delta$ spa47 mutant strain BS652. Analysis of BS103 and BS652 revealed that both the chromosome and virulence plasmid encode enterotoxins. E. coli $\mathrm{HS}$ served as a negative control. Experiments were performed with intestinal tissue from rabbits (A) and mice (B). All differences are significant, with $p$-values $<0.01$.

doi:10.1371/journal.pone.0049980.g003

Analysis of the secreted proteins from these strains demonstrated that enterotoxic activity was still present above E. coli HS levels (Figure 7A). These observations confirmed that S. flexneri encoded additional enterotoxins; and therefore, we proceeded to analyze a $\triangle \operatorname{sep} A$ mutant generously provided by Dr. Beth McCormick. SepA is a Serine Protease Autotransporter of Enterobacteriacae (SPATE) that is encoded on the virulence plasmid [31]. This protein does not require the T3SS to be secreted, and therefore, served as a likely candidate to be the additional enterotoxin encoded on the virulence plasmid. The $\Delta$ sepA mutant elicited a $\Delta$ Isc significantly lower than wildtype (Figure $7 \mathrm{~A}$ ), indicating that SepA contributed to the enterotoxic activity.

\section{Discussion}

The mechanism by which Shigella causes watery diarrhea is currently unknown. Since secretogenic activity is a central hallmark of early disease progression, the goal of this study was to expand previous work on Shigella-induced secretion through the validation of a mouse jejunum model using Ussing chambers. By analyzing several $S$. flexneri mutants, we were able to characterize

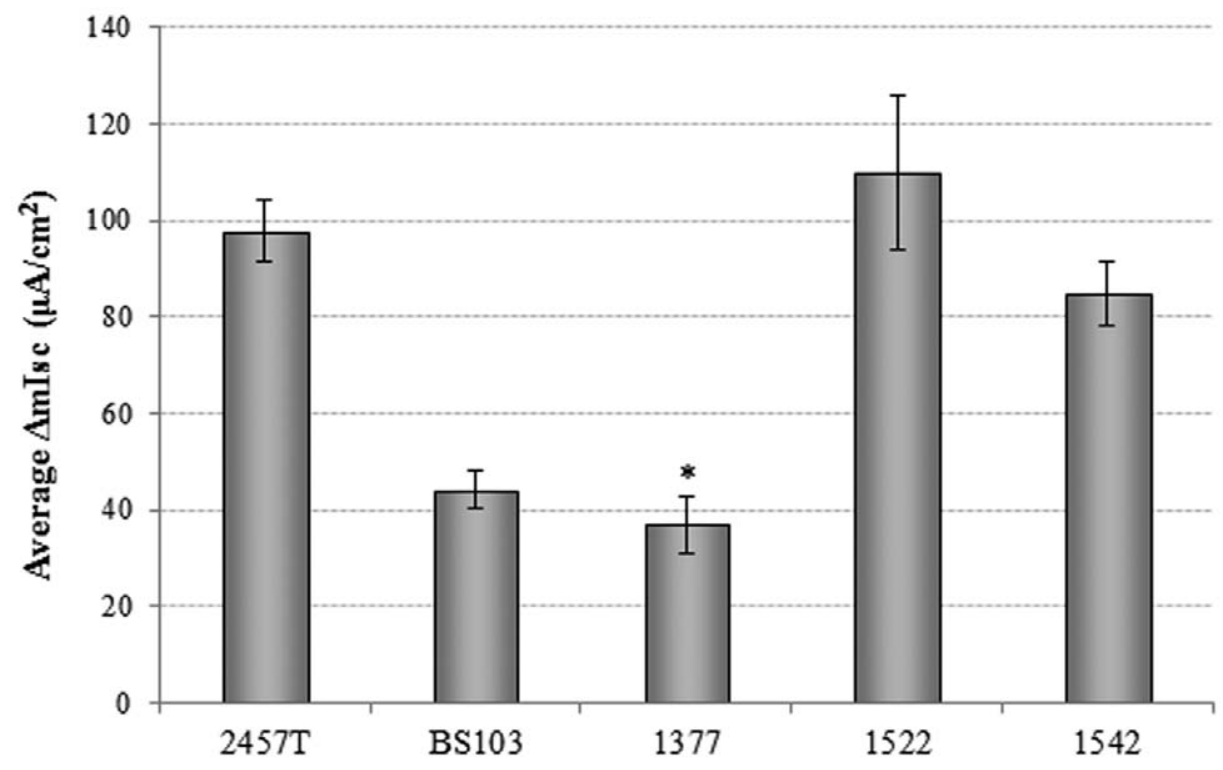

Figure 4. The pic and set $1 A B$ locus encodes the chromosomal toxins. Strain 1377 ( $\triangle p i c+\Delta s e t 1 A B)$ had almost a $60 \%$ reduction in the change in short circuit current compared to wildtype 2457T. Complementation of the mutation in strain 1522 restores toxin activity to the same level as 2457T. In addition, addition of pic alone in strain 1542 restores $\Delta \mathrm{Isc}$ to the same level as wildtype bacteria. The $p$-value difference between $2457 \mathrm{~T}$ and 1377 is $<0.001$, as is the difference between 1377 and 1522 or 1542 . There was no significant difference between $2457 T, 1522$, or 1542 . doi:10.1371/journal.pone.0049980.g004 


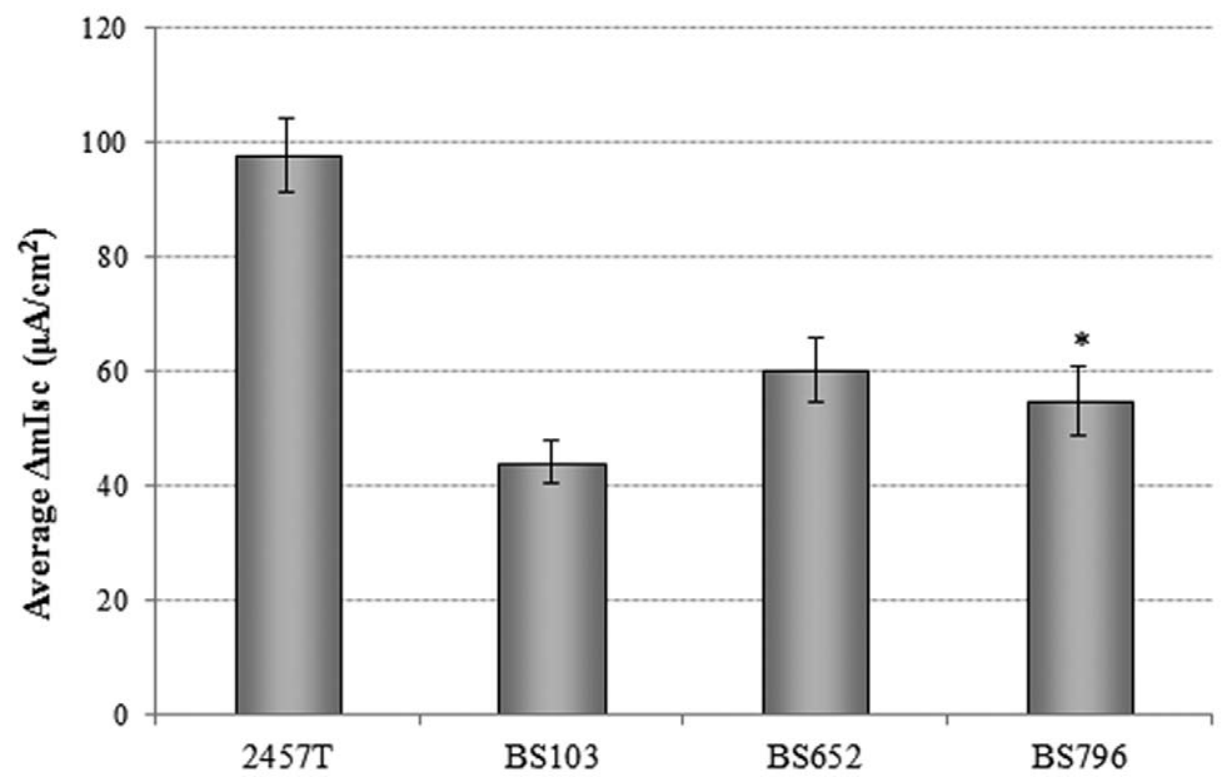

Figure 5. The type-III secretion system effector ShET2 (encoded by sen or ospD3) contributes to enterotoxin activity. A mutant that does not express ShET2 (BS796) causes the same change in short circuit current as the type-III secretion system mutant strain BS652. The $p$-value difference between 2457T and BS796 is $<0.01$. The data indicate that ShET2 is the type-III secretion system effector protein that contributes to enterotoxin activity.

doi:10.1371/journal.pone.0049980.g005

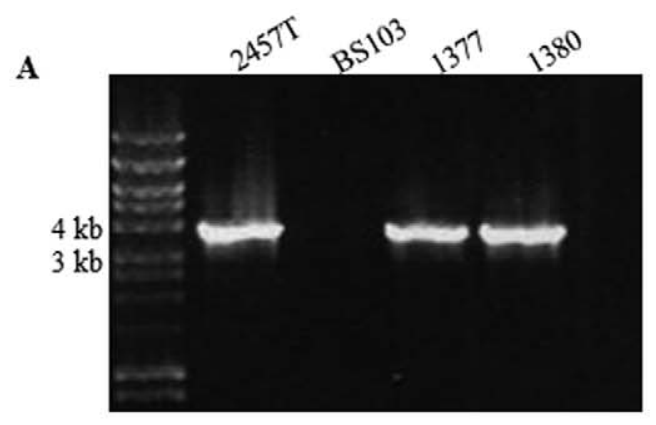

B
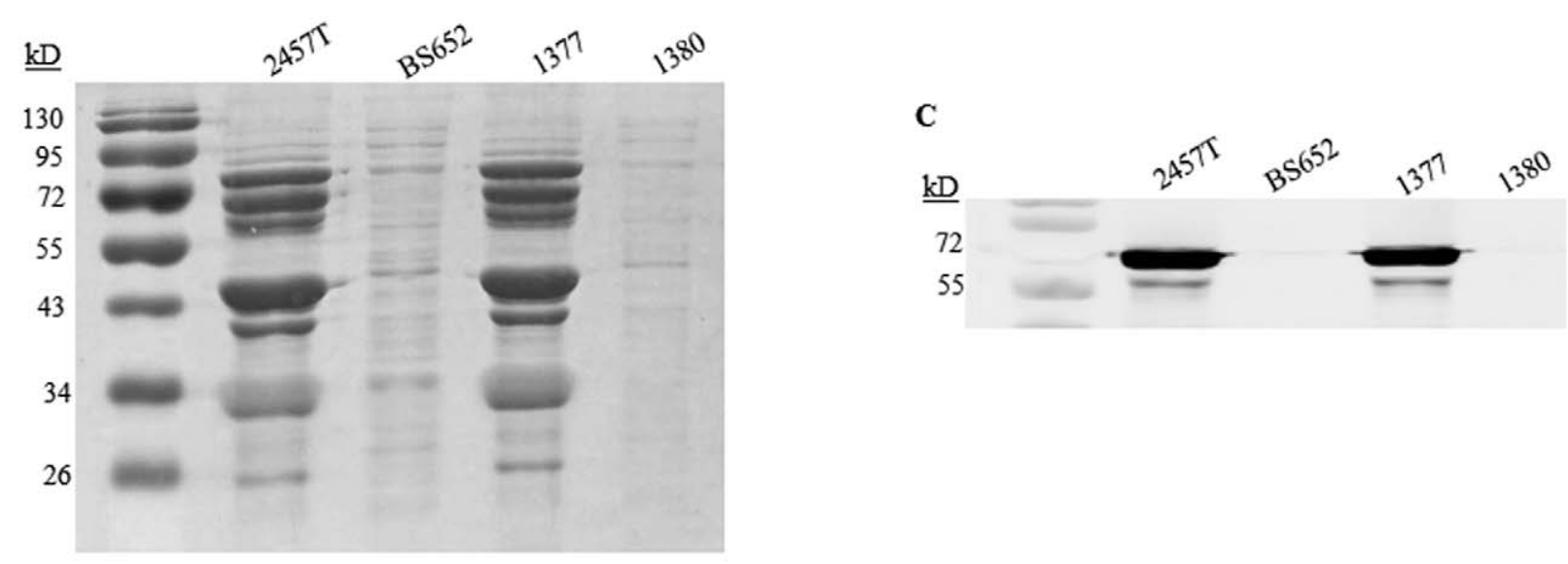

Figure 6. Strain 1380 has the virulence plasmid but a spontaneous mutation results in a defective T3SS. A: PCR analysis for the ospC1 to ospD3 coding region ( $3.7 \mathrm{~kb})$ demonstrates presence of the virulence plasmid. The negative control is the plasmid-cured strain BS103. B: The Congo red secretion assay was performed, and the supernatants representing the secreted proteins were analyzed on an SDS-PAGE gel. The secretion profile of 2457T, BS652, 1377, and 1380 demonstrates that 1380 had a dysfunctional T3SS like the $\Delta$ spa47 mutant (BS652). C: Western blot analysis of supernatants for IpaB. Secretion was only detected in the strains with a functional T3SS, strains 2457T and 1377.

doi:10.1371/journal.pone.0049980.g006 
A

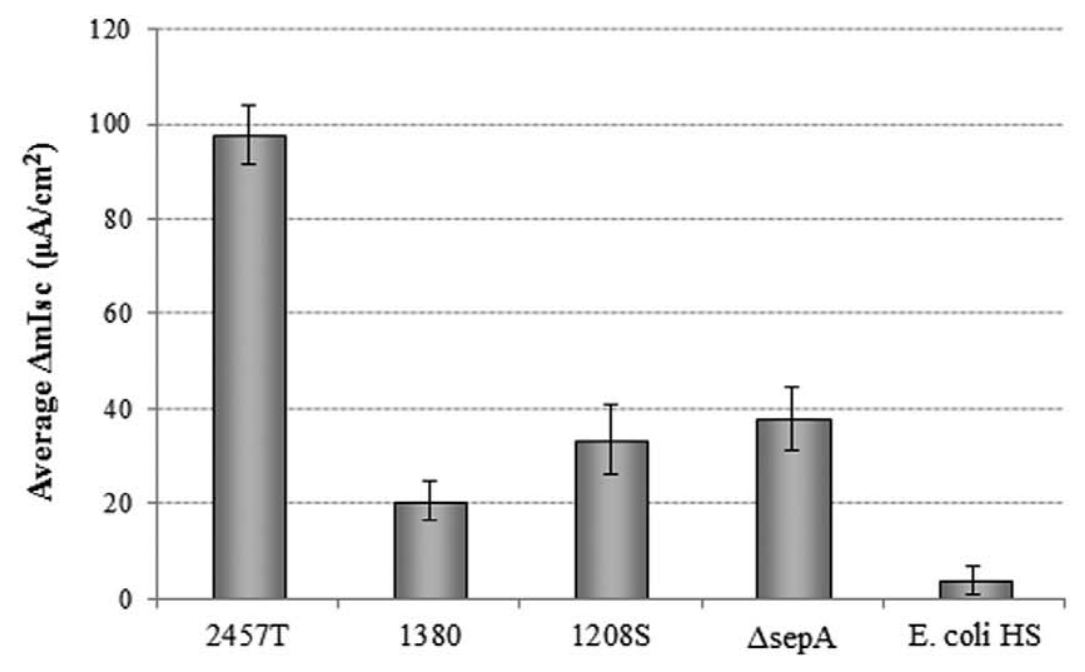

B

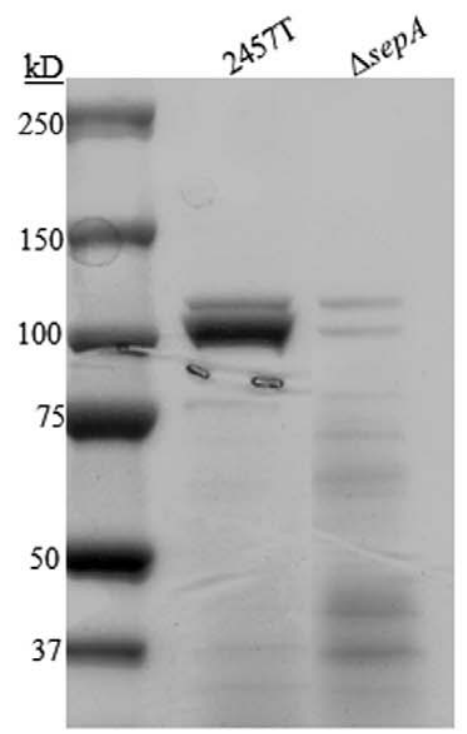

Figure 7. Additional Shigella proteins contribute to enterotoxin activity. A: Analysis of 1380 and $1208 S$ verified that S. flexneri encodes enterotoxins in addition to Pic, ShET1, and ShET2. Analysis of a $\triangle$ sepA mutant demonstrated there was a significant decrease in the short circuit current, indicating that SepA contributed to enterotoxic activity. All 3 mutant strains have a significant increase in $\triangle \mathrm{Isc}$ compared to $E$. coli HS with all $p$-values $<0.01$. B: Coomassie-stained protein gel of the secreted proteins obtained from the $\Delta$ sepA mutant after growth and concentration of cultural supernatants. SepA represents a large portion of the high molecular weight autotransporter proteins. doi:10.1371/journal.pone.0049980.g007

the genomic determinants encoding toxins capable of increasing intestinal secretion signals. Previous studies in the field were aimed at characterizing ShET1 and ShET2 as Shigella enterotoxins but did not extend the study to address the presence of additional toxins. In the previous studies, the toxins were cloned and artificially expressed in E. coli, and the purified proteins were applied to rabbit jejunum mounted in Ussing chambers $[4,8,9]$. While this approach was important to demonstrate that toxin addition alone was sufficient to induce changes in Isc, the higher level of protein expressed and possible alterations from production in $E$. coli could affect the amount of short circuit change detected. By using the natural host, our studies with $S$. flexneri mutants allowed us to dissect the specific contribution of each enterotoxin previously described (ShET1 and ShET2) and of those newly discovered by our studies (Pic and SepA) in causing Shigella electrolyte secretion in the small intestine. The use of the natural host can also address potential interactions that occur between toxins and other secreted proteins.

A major advance, and a central aspect of our studies compared to previous reports, was the utilization of mouse tissue in the Ussing chamber experiments. We chose $\mathrm{C} 57 \mathrm{BL} / 6$ mice as we will perform additional studies to address the roles of these toxins in knockout mice on the same genetic background lacking specific pattern recognition pathways or secretory pathways to gain more insights on the mechanism of action Shigella-induced secretory diarrhea. In addition, female mice have less of a tendency to fight, which is important since fighting can affect the immune system and other bodily systems. For these reasons this strain of mice was ideal for our studies. We have validated this model against the previously reported rabbit model in order to use mouse intestinal tissue as a reliable and efficient assay for our current and future studies. Our results demonstrate that mouse jejunum in the Ussing chamber system is as responsive to Shigella toxins as rabbit jejunum. Although several models of shigellosis have been developed, including the nonhuman primate model, the guinea pig keratoconjunctivitis model, and the pulmonary shigellosis model, the models do not adequately measure the watery diarrheal phase of infection $[32,33]$. This study is the first to demonstrate secretogenic reactivity in an ex vivo mouse jejunum model to Shigella toxins.

The finding that the iron-depleted conditions were not necessary to study Shigella enterotoxic activities is an important aspect of this study. The iron-depleted medium that was initially utilized was based on the fact that these conditions were required to detect a change in Isc in studies with enteroinvasive E. coli [21]. Given the similarity between EIEC and Shigella, the iron-depleted conditions were continued into the Shigella studies $[4,8,9]$. In addition, supernatants isolated from a plasmid-cured strain of $S$. flexneri induced higher levels of water accumulation in the rabbit ileal loop model when the cultures were grown in the absence of iron [8]. However, another bacterial factor present in the culture supernatants, not ShET1, could have been affected by the iron limitation and enhanced water accumulation in the rabbit intestine. The data presented in figure 1 clearly demonstrate that iron-depletion is not required for Ussing chamber analysis of the Shigella enterotoxins. Whether iron limitation affects the efficacy of other Shigella proteins in the rabbit ileal loop analyses remains to be determined. Of course, use of other culture conditions could yield still more enterotoxins that may be produced in vivo.

Our mutant analysis strategy was instrumental in this study. We specifically chose to compare the plasmid-cured strain (BS103) and the $\Delta$ spa 47 mutant (BS652), a T3SS mutant that has been shown to be incapable of secreting T3SS effectors [24,25]. Based on the secretion profile in figure 2 , the autotransporters encoded on the virulence plasmid were still secreted in this mutant. In recent years three proteins belonging to the Serine Protease Autotransporter of Enterobacteriacae (SPATE) family have been identified in S. flexneri: SepA, SigA, and Pic. SepA is non-T3SS effector encoded on the virulence plasmid. Although the substrate(s) of SepA has not been identified, a recent study associated SepA with diarrheal cases in 
Mali [34]. SigA is encoded in the she pathogenicity island, and contributes to secretion in the rabbit ileal loop model [27]. AlHasani et al., recently defined the structural protein fodrin as a target of SigA, implicating a role for this SPATE in cytoskeletal reorganization [35]. Finally, Pic is a mucinase that is encoded on the DNA strand directly opposite of the genes for ShET1. A recent study defined the substrate specificity of Pic, an immune system modulator [16]. Given the presence of the autotransporters in the culture supernatants and the association of these factors with diarrheal disease, we hypothesized that the SPATES could also contribute to enterotoxin activity. We therefore proceeded with our mutational analysis to determine if these SPATES had a role in inducing secretogenic activity.

Initially, analysis of BS103 and BS652 allowed us to determine if toxins were encoded on the chromosome and/or virulence plasmid. In addition, use of the $\Delta$ spa 47 mutant allowed us to demonstrate that the virulence plasmid harbored toxins that were T3-dependent and T3-independent since there was a significant increase in Isc compared to the plasmid-cured mutant (BS103) (figure 3). Based on our subtraction analysis, we conclude that three groups of toxins contribute to the increase in Isc: one group on the chromosome, one group on the virulence plasmid that requires the T3SS, and one group on the virulence plasmid that is secreted independently of the T3SS.

Further analysis indicated that the ShET1/Pic region is a major contributor of enterotoxin activity from the chromosome, which was confirmed by the analysis of the complementation of strain 1377 with ShET1-containing 1522 and ShET1-devoid 1542. Pic is transcribed on the opposite strand of DNA from the genes encoding ShET1, is only present in S. flexneri, and has been shown to target a wide range of leukocyte adhesion proteins [16]. How Pic contributes to secretogenic activity in our system and in vivo remains to be elucidated, and could function indirectly as an enterotoxin by affecting another protein. It is unclear in the previous literature whether or not the entire pic gene was present in the experiments in which the genes encoding ShET1 were cloned for expression and purification, but purified ShET1 has been shown to induce secretion in the rabbit ileal loop model $[8,9]$. Our studies indicate that the majority of the enterotoxic activity present in Shigella strain 2457T is due to Pic.

Comparison of BS103 and the $\Delta$ spa47 mutant indicated the presence of an additional factor encoded on the virulence plasmid that affected Isc. The $\Delta o s p D 3$ mutant analysis confirmed that ShET2 is the T3SS effector responsible for enterotoxin activity, which agrees with previous literature [4]. In fact, Nataro et al. hypothesized that ShET2 was a T3SS effector protein since a signal peptide could not be detected [4]. Analysis of strains $1208 \mathrm{~S}$ and 1380 also indicated there were additional enterotoxins encoded by $S$. flexneri. We chose to analyze a $\Delta s e p A$ mutant since it

\section{References}

1. Weisz A, Meuli G, Thakwalakwa C, Trehan I, Maleta K, et al. (2011) The duration of diarrhea and fever is associated with growth faltering in rural Malawian children aged 6-18 months. Nutr J 10: 25.

2. O'Ryan M, Prado V, Pickering LK (2005) A millennium update on pediatric diarrheal illness in the developing world. Semin Pediatr Infect Dis 16: 125-136.

3. Kotloff KL, Winickoff JP, Ivanoff B, Clemens JD, Swerdlow DL, et al. (1999) Global burden of Shigella infections: implications for vaccine development and implementation of control strategies. Bull World Health Organ 77: 651-666.

4. Nataro JP, Seriwatana J, Fasano A, Maneval DR, Guers LD, et al. (1995) Identification and cloning of a novel plasmid-encoded enterotoxin of enteroinvasive Escherichia coli and Shigella strains. Infect Immun 63: 4721-4728.

5. Farfan MJ, Toro CS, Barry EM, NataroJP (2011) Shigella enterotoxin-2 is a type III effector that participates in Shigella-induced interleukin 8 secretion by epithelial cells. FEMS Immunol Med Microbiol 61: 332-339.

6. Henderson IR, Nataro JP (2001) Virulence functions of autotransporter proteins. Infect Immun 69: 1231-1243. is an autotransporter encoded on the virulence plasmid that has been shown to affect fluid accumulation in rabbit ileal loops [31] and to contribute to diarrhea in enteroaggregative $E$. coli [34]. The $\Delta$ sepA mutant caused a significant drop in Isc compared to wildtype bacteria (Figure 7A). The data demonstrate that SepA has enterotoxic activity; however, there may be two reasons as to why the mutation caused such a dramatic drop in Isc compared to wildtype bacteria. First, analysis of the concentrated proteins from the mutant demonstrates that a significant amount of SepA is secreted into the cultures (Figure 7B). Second, SepA may target the same eukaryotic protein or pathway as another enterotoxin to enhance the overall toxin effect. Given the fact that in our culture conditions the $\Delta$ spa47 mutant had an approximate $20 \%$ increase in Isc compared to BS103, and that strain 1380 (with only SepA present) had approximately $20 \%$ increase compared to the negative control $E$. coli $\mathrm{HS}$, we believe that the SepA contribution is approximately $20 \%$ in our system. As with Pic, SepA could function indirectly as an enterotoxin by affecting another protein. Further characterization of SepA will determine the role of this autotransporter protein in our system and in vivo.

In conclusion, our study confirms the enterotoxin activity of ShET1 and ShET2 from previous publications, validates the use of mouse jejunum in Ussing chamber analyses as a novel, more versatile and high-throughput bioassay for Shigella enterotoxic activity, and identifies additional Shigella proteins (Pic and SepA) that contribute to enterotoxin activity. The results obtained in this study may assist future studies for identification of eukaryotic protein targets. The fact that we have yet to identify a toxin-null mutant indicates there may be additional enterotoxin(s) encoded by $S$. flexneri that contribute to the overall watery component of Shigella-induced diarrhea. Despite this possibility, we are confident that we have demonstrated that ShET1/Pic, ShET2, and SepA are the factors that contribute most prominently to the changes in Isc in mouse jejunum in the Ussing chambers.

\section{Acknowledgments}

The authors would like to thank Dr. Anthony Maurelli, Uniformed Services University, and Dr. Beth McCormick, University of Massachusetts Medical School, for the bacterial strains used in this study. The authors would also like to thank Dr. Edwin Oaks, Walter Reed Army Institute of Research, for the IpaB antibody used in this study.

\section{Author Contributions}

Conceived and designed the experiments: CF JH JK AF JN. Performed the experiments: CF JH TSD. Analyzed the data: CF JH AF JN. Contributed reagents/materials/analysis tools: TSD EB AF JN. Wrote the paper: $\mathrm{CF}$ JH AF JPN.

7. Schiavo G, van der Goot FG (2001) The bacterial toxin toolkit. Nat Rev Mol Cell Biol 2: 530-537.

8. Fasano A, Noriega FR, Maneval DR, Jr., Chanasongcram S, Russell R, et al. (1995) Shigella enterotoxin 1: an enterotoxin of Shigella flexneri 2a active in rabbit small intestine in vivo and in vitro. J Clin Invest 95: 2853-2861.

9. Fasano A, Noriega FR, Liao FM, Wang W, Levine MM (1997) Effect of Shigella enterotoxin 1 (ShET1) on rabbit intestine in vitro and in vivo. Gut 40: 505-511.

10. Farfan MJ, Garay TA, Prado CA, Filliol I, Ulloa MT, et al. (2010) A new multiplex PCR for differential identification of Shigella flexneri and Shigella sonnei and detection of Shigella virulence determinants. Epidemiol Infect 138: 525-533.

11. Niyogi SK (2005) Shigellosis. J Microbiol 43: 133-143.

12. Schroeder GN, Hilbi H (2008) Molecular pathogenesis of Shigella spp.: controlling host cell signaling, invasion, and death by type III secretion. Clin Microbiol Rev 21: 134-156. 
13. Takeuchi A, Formal SB, Sprinz H (1968) Exerimental acute colitis in the Rhesus monkey following peroral infection with Shigella flexneri. An electron microscope study. Am J Pathol 52: 503-529.

14. Clarke LL (2009) A guide to Ussing chamber studies of mouse intestine. Am J Physiol Gastrointest Liver Physiol 296: G1151-1166.

15. Datsenko KA, Wanner BL (2000) One-step inactivation of chromosomal genes in Escherichia coli K-12 using PCR products. Proc Natl Acad Sci U S A 97: 66406645 .

16. Ruiz-Perez F, Wahid R, Faherty CS, Kolappaswamy K, Rodriguez L, et al. (2011) Serine protease autotransporters from Shigella flexneri and pathogenic Escherichia coli target a broad range of leukocyte glycoproteins. Proc Natl Acad Sci U S A. 108: 12881-12886.

17. Maurelli AT, Curtiss R, 3rd (1984) Bacteriophage Mu dl(Apr lac) generates virlac operon fusions in Shigella flexneri 2a. Infect Immun 45: 642-648.

18. Sutton TL, Zhao A, Madden KB, Elfrey JE, Tuft BA, et al. (2008) AntiInflammatory mechanisms of enteric Heligmosomoides polygyrus infection against trinitrobenzene sulfonic acid-induced colitis in a murine model. Infect Immun 76: $4772-4782$.

19. Bahrani FK, Sansonetti PJ, Parsot G (1997) Secretion of Ipa proteins by Shigella flexneri: inducer molecules and kinetics of activation. Infect Immun 65: 40054010.

20. Mills JA, Buysse JM, Oaks EV (1988) Shigella flexneri invasion plasmid antigens B and $\mathrm{C}$ : epitope location and characterization with monoclonal antibodies. Infect Immun 56: 2933-2941.

21. Fasano A, Kay BA, Russell RG, Maneval DR, Jr., Levin MM (1990) Enterotoxin and cytotoxin production by enteroinvasive Escherichia coli. Infect Immun 58: 3717-3723.

22. Burleigh DE, Borman RA (1997) Evidence for a nonneural electrogenic effect of cholera toxin on human isolated ileal mucosa. Dig Dis Sci 42: 1964-1968.

23. Levine MM, Bergquist EJ, Nalin DR, Waterman DH, Hornick RB, et al. (1978) Escherichia coli strains that cause diarrhoea but do not produce heat-labile or heatstable enterotoxins and are non-invasive. Lancet 1: 1119-1122.

24. Tamano K, Aizawa S, Katayama E, Nonaka T, Imajoh-Ohmi S, et al. (2000) Supramolecular structure of the Shigella type III secretion machinery: the needle part is changeable in length and essential for delivery of effectors. EMBO J 19: 3876-3887.

25. Venkatesan MM, Buysse JM, Oaks EV (1992) Surface presentation of Shigella flexneri invasion plasmid antigens requires the products of the spa locus. J Bacteriol 174: 1990-2001.

26. Benjelloun-Touimi Z, Si Tahar M, Montecucco C, Sansonetti PJ, Parsot C (1998) SepA, the $110 \mathrm{kDa}$ protein secreted by Shigella flexneri: two-domain structure and proteolytic activity. Microbiology 144 (Pt 7): 1815-1822.
27. Al-Hasani K, Henderson IR, Sakellaris H, Rajakumar K, Grant T, et al. (2000) The sigA gene which is borne on the she pathogenicity island of Shigella flexneri 2a encodes an exported cytopathic protease involved in intestinal fluid accumulation. Infect Immun 68: 2457-2463.

28. Henderson IR, Czeczulin J, Eslava C, Noriega F, Nataro JP (1999) Characterization of pic, a secreted protease of Shigella flexneri and enteroaggregative Escherichia coli. Infect Immun 67: 5587-5596.

29. Wagner JK, Heindl JE, Gray AN, Jain S, Goldberg MB (2009) Contribution of the periplasmic chaperone Skp to efficient presentation of the autotransporter IcsA on the surface of Shigella flexneri. J Bacteriol 191: 815-821.

30. Le Gall T, Mavris M, Martino MC, Bernardini ML, Denamur E, et al. (2005) Analysis of virulence plasmid gene expression defines three classes of effectors in the type III secretion system of Shigella flexneri. Microbiology 151: 951-962.

31. Benjelloun-Touimi Z, Sansonetti PJ, Parsot C (1995) SepA, the major extracellular protein of Shigella flexneri: autonomous secretion and involvement in tissue invasion. Mol Microbiol 17: 123-135.

32. Barman S, Saha DR, Ramamurthy T, Koley H (2011) Development of a new guinea-pig model of shigellosis. FEMS Immunol Med Microbiol 62: 304-314.

33. Pore D, Mahata N, Pal A, Chakrabarti MK (2011) Outer membrane protein A $(\mathrm{OmpA})$ of Shigella flexneri $2 \mathrm{a}$, induces protective immune response in a mouse model. PLoS One 6: e22663.

34. Boisen N, Scheutz F, Rasko DA, Redman JC, Persson S, et al. (2012) Genomic characterization of enteroaggregative Escherichia coli from children in Mali. J Infect Dis 205: 431-444.

35. Al-Hasani K, Navarro-Garcia F, Huerta J, Sakellaris H, Adler B (2009) The immunogenic SigA enterotoxin of Shigella flexneri 2 a binds to HEp-2 cells and induces fodrin redistribution in intoxicated epithelial cells. PLoS One 4: e8223.

36. Formal SB, Dammin GJ, Labrec EH, Schneider H (1958) Experimental Shigella infections: characteristics of a fatal infection produced in guinea pigs. J Bacteriol 75: 604-610.

37. Maurelli AT, Blackmon B, Curtiss R, 3rd (1984) Loss of pigmentation in Shigella flexneri $2 \mathrm{a}$ is correlated with loss of virulence and virulence-associated plasmid. Infect Immun 43: 397-401.

38. Faherty CS, Maurelli AT (2009) Spa15 of Shigella flexneri is secreted through the type III secretion system and prevents staurosporine-induced apoptosis. Infect Immun 77: 5281-5290.

39. Kotloff KL, Simon JK, Pasetti MF, Sztein MB, Wooden SL, et al. (2007) Safety and immunogenicity of CVD 1208S, a live, oral DeltaguaBA Deltasen Deltaset Shigella flexneri $2 \mathrm{a}$ vaccine grown on animal-free media. Hum Vaccin 3: 268-275. 